\title{
GENERALIZED INTERPOLATION IN THE UNIT BALL
}

\author{
NicOlas MARCO
}

\begin{abstract}
We study a generalized interpolation problem for the space $H^{\infty}\left(\mathbb{B}^{2}\right)$ of bounded homomorphic functions in the ball $\mathbb{B}^{2}$. A sequence $Z=\left\{z_{n}\right\}$ of $\mathbb{B}^{2}$ is an interpolating sequence of order 1 if for all sequence of values $w_{n}$ satisfying conditions of order 1 (that is discrete derivatives in the pseudohyperbolic metric are bounded) there exists a function $f \in H^{\infty}\left(\mathbb{B}^{2}\right)$ such that $f\left(z_{n}\right)=w_{n}$. These sequences are characterized as unions of 3 free interpolating sequences for $H^{\infty}\left(\mathbb{B}^{2}\right)$ such that all triplets of $Z$ made of 3 nearby points have to define an angle uniformly bounded below (in an appropriate sense). Also, we give a multiple interpolation result (interpolation of values and derivatives).
\end{abstract}

\section{Introduction}

Let $\mathbb{B}=\mathbb{B}^{n}$ be the unit ball of $\mathbb{C}^{n}, Z=\left(z_{k}\right)_{k \in \mathbb{N}}$ a sequence of $\mathbb{B}$, and $H^{\infty}(\mathbb{B})=\mathcal{O}(\mathbb{B}) \cap L^{\infty}(\mathbb{B}):=\left\{f \in \mathcal{O}(\mathbb{B})\right.$ s.t. $\|f\|:=\sup _{z \in \mathbb{B}}|f(z)|<$ $\infty\}$ the space of all bounded holomorphic functions in $\mathbb{B}$. We shall say that $Z$ is a free interpolating sequence for $H^{\infty}(\mathbb{B})$ if for all sequence of values $\left(w_{k}\right)_{k \in \mathbb{N}} \in \ell^{\infty}$ there exists $f \in H^{\infty}(\mathbb{B})$ such that $f\left(z_{k}\right)=w_{k}$ for all $k \in \mathbb{N}$ (we write $Z \in \operatorname{Int}_{0}\left(H^{\infty}(\mathbb{B})\right)$ ).

When $n=1$, let $\mathbb{D}=\mathbb{B}^{1}$ be the unit disk of $\mathbb{C}$. Free interpolating sequences for $H^{\infty}(\mathbb{D})$ were characterized by L. Carleson [Car58] in terms of Blaschke products. Also, this characterization can be extended to interpolating sequences for the Hardy spaces $H^{p}(\mathbb{D})$ of the disk (see H. S. Shapiro, A. L. Shields [SS61]).

In [Vas79], [Nik78a], [Nik78b], [BNØ96], [Har99], [Mar98], several authors have studied a new kind of interpolation where one takes into account dependency between the values to be interpolated at neighbouring points. We call this type of interpolation generalized interpolation. More precisely in [BNØ96] and [Mar98] the authors obtained a characterization of generalized interpolation in term of union of free

2000 Mathematics Subject Classification. 30E05.

Key words. Generalized interpolation. 
interpolating sequences. The goal of this paper is to give such results for several variables.

Notations. Let $\phi_{a}$ be the unique involutive automorphism of $\mathbb{B}$ exchanging the point $a$ and 0 , and denote $\rho(z, \xi):=\left|\phi_{z}(\xi)\right|$ the pseudohyperbolic distance between $z$ and $\xi$. Also we will use the notation $K(z, r)$ for the pseudohyperbolic ball of center $z$ and radius $r$. (This ball is similar to an ellipsoid of center $z$ and radius $r\left(1-|z|^{2}\right)$ in the direction of $z$ and radius $r\left(1-|z|^{2}\right)^{1 / 2}$ in the "tangential" directions orthogonal to $z$.)

We say that a sequence $Z$ of $\mathbb{B}$ is separated if $m:=\inf _{k \neq j} \rho\left(z_{k}, z_{j}\right)>0$. We then call $m$ the separation constant.

For $z, \xi \in \mathbb{C}^{n},\langle z, \xi\rangle=\sum_{i=1}^{n} z_{i} \bar{\xi}_{i}$ denotes the usual scalar product of $\mathbb{C}^{n}$, and $\mathcal{A}(z, \xi)$ will denote the value of the angle between $z$ et $\xi$, defined by

$$
\mathcal{A}(z, \xi)=\arccos \frac{|\langle z, \xi\rangle|}{|z||\xi|} .
$$

Also, for $a, b \in \mathbb{B}$, we will use the Kronecker symbol $\delta_{a}^{b}:=\left\{\begin{array}{ll}1 & \text { if } a=b \\ 0 & \text { otherwise }\end{array}\right.$.

When $n>1$ we don't know any characterization for free interpolating sequences, but some necessary and sufficiency conditions are available. N. Th. Varopoulos [Var72] obtained a necessary condition for $H^{\infty}$ free interpolation:

Theorem (N. Th. Varopoulos [Var72]). Let $Z$ be a free interpolating sequence in $\mathbb{B}^{n}$ for $H^{\infty}\left(\mathbb{B}^{n}\right)$ then $Z$ is a separated sequence, and $\sup _{k \in \mathbb{N}} \sum_{j=0}^{\infty}\left(1-\rho^{2}\left(z_{j}, z_{k}\right)\right)^{n}<\infty$.

This necessary condition is far from sufficient and P. J. Thomas [Tho97] gave other necessary conditions implying Varopoulos's condition. Also, we have a sufficient condition:

Theorem (B. Berndtsson [Ber85]). Let $Z$ be a separated sequence of $\mathbb{B}^{n}$ such that $\sup _{k \in \mathbb{N}} \sum_{j=0}^{\infty}\left(1-\rho^{2}\left(z_{j}, z_{k}\right)\right)<\infty$, then $Z$ is a free interpolating sequence for $H^{\infty}\left(\mathbb{B}^{n}\right)$.

To define generalized interpolation, let us specify a new space of values:

$$
\begin{aligned}
\mathcal{V}^{1}(Z)=\left\{w=\left(w_{k}\right)_{k \in \mathbb{N}}\right. \text { such that } \\
\left.\qquad\left.|| w\right|_{\mathcal{V}^{1}(Z)}:=\max \left(\sup _{k \neq j} \frac{\left|w_{k}-w_{j}\right|}{\rho\left(z_{k}, z_{j}\right)}, \sup _{k \in \mathbb{N}}\left|w_{k}\right|\right)<\infty\right\} .
\end{aligned}
$$


Definition 1. The sequence $Z$ is called an interpolating sequence of order 1 for $H^{\infty}(\mathbb{B})$ if for all $w \in \mathcal{V}^{1}(Z)$ there exists $f \in H^{\infty}(\mathbb{B})$ such that $f_{\mid Z}=w$. We write $Z \in \operatorname{Int}_{1}\left(H^{\infty}(\mathbb{B})\right)$.

The notion of interpolating sequence can be expressed in terms of a restriction operator. Let

$$
\begin{aligned}
R_{Z}: H^{\infty}(\mathbb{B}) & \longrightarrow \mathbb{C}^{\mathbb{N}} \\
f & \longrightarrow\left(f\left(z_{k}\right)\right)_{k \in \mathbb{N}},
\end{aligned}
$$

then $Z$ is an interpolating sequence of order 1 iff $R_{Z}$ is onto $\mathcal{V}^{1}(Z)$. Note that if that is the case, an argument based on the open mapping theorem shows that interpolation can be performed with control of the norm of the interpolating function. There exists a constant $M$ such that for all $W \in \mathcal{V}^{1}(Z)$, there exists $f \in H^{\infty}(\mathbb{B})$ such that $R_{Z}(f)=W$ and $\|f\| \leq M\|W\|$. The smallest constant $M$ satisfying this is called the interpolating constant of order 1 of $Z$, we will denote it by $M_{1}(Z)$.

The interpolating sequences of order 1 have been characterized for the case of the disk in [Mar98] as sequences which are the union of 2 free interpolating sequences for $H^{\infty}$. More generally, we can, in one variable, extend the result by defining a space of values $\mathcal{V}^{k}$ which control discrete derivatives (pseudohyperbolic divided differences) up to order $k$ (instead of order 1). We then obtain that $Z$ is an interpolating sequence of order $k$ iff $Z$ is the union of $k+1$ free interpolating sequences for $H^{\infty}$. Also, these results have a meaning for $p<\infty$ (see [BNØ96], [Mar98]).

In several variables, divided differences of order greater than 1 and such tools as the Jensen formula or Blaschke products are not available. For those reasons we restrict the study to $H^{\infty}\left(\mathbb{B}^{2}\right)$ and order 1 . We obtain

Theorem 2. $Z$ is an interpolating sequence of order 1 if and only if

(i) $Z$ is the union of at most 3 free interpolating sequences $A, B, C$.

(ii) There exist $\gamma>0$ and $\theta>0$ such that for all $a \in A, b \in B, c \in C$, satisfying $b, c \in K(a, \gamma)$, we have $\mathcal{A}\left(\phi_{a}(b), \phi_{a}(c)\right) \geq \theta$.

Remark 3. It is surprising to obtain an "if and only if" result, particularly so as no description of free interpolating sequences is available.

This theorem may remain true for dimensions $n>2$ but at the price of much technical complication. We would obtain a condition such as: $Z$ is a union of $n+1$ separated sequences, and any $n+1$-tuple made up of $n+1$ nearby points has, after application of an automorphism and normalisation, a system of $n$ vectors with a determinant uniformly bounded below. 
For higher order the problem is harder since we don't have finite differences of higher order to define $\mathcal{V}^{k}(Z)$ for $k \geq 2$ and it is a good question to find how it is possible to formulate this problem.

We will also obtain a multiple interpolation result: let $\mathcal{L}_{i}\left(\mathbb{C}^{2}\right)$ be the space of all symmetric multilinear maps from $\left(\mathbb{C}^{2}\right)^{i}$ to $\mathbb{C}$, and

$\mathcal{M}^{i}(Z)=\left\{\left(l_{k}^{(j)}\right)_{k \in \mathbb{N}}, j=0, \ldots, i, l_{k}^{(j)} \in \mathcal{L}_{j}\left(\mathbb{C}^{2}\right), \sup _{\substack{k \in \mathbb{N} \\ j=0, \ldots, i}}\left|\left\|l_{k}^{(j)} \mid\right\|<\infty\right\}\right.$,

where ||I.|| designs the usual norm of a multilinear map.

Definition 4. The sequence $Z$ is a multiple interpolating sequence of order $i$ if for all $\left(l_{k}^{(j)}\right)_{k \in \mathbb{N}} \in \mathcal{M}^{i}(Z)$, there exists $f \in H^{\infty}(\mathbb{B})$ such that $D^{j}\left(f \circ \phi_{z_{k}}\right)(0)=l_{k}^{(j)}$ for all $k \in \mathbb{N}, j=0, \ldots, i$.

Clearly, any multiple interpolating sequence of order $i$ is interpolating of order 0 (that is free interpolating). The converse actually holds.

Theorem 5. The sequence $Z$ is a free interpolating sequence if and only if $Z$ is a multiple interpolating sequence of arbitrary order.

The present work is part of the author's $\mathrm{Ph}$. D. dissertation, and he thanks his advisor Pascal Thomas for his help during its completion.

\section{Preliminaries}

It is clear from the definition that a subsequence of a free interpolating sequence is still a free interpolating sequence. For interpolation of order 1, it remains true, although not so obvious. It follows from

Proposition 6. Let $Z=\left(z_{q}\right)_{q \in I}$ a sequence of $\mathbb{B}$ and $Z^{\prime}=\left(z_{q}\right)_{q \in I^{\prime}}$ a subsequence of $Z$. For all $w=\left(w_{q}\right)_{q \in I^{\prime}} \in \mathcal{V}^{1}\left(Z^{\prime}\right)$ there exists $\left(\tilde{w}_{q}\right)_{q \in I} \in$ $\mathcal{V}^{1}(Z)$ an extension of $w$ satisfying

$$
\|\tilde{w}\|_{\mathcal{V}^{1}(Z)} \leq \sqrt{2}\|w\|_{\mathcal{V}^{1}\left(Z^{\prime}\right)} .
$$

We conclude immediately that if $Z \in \operatorname{Int}_{1}\left(H^{\infty}(\mathbb{B})\right)$ then $Z^{\prime} \in \operatorname{Int}_{1}\left(H^{\infty}(\mathbb{B})\right)$. Indeed, let $w \in \mathcal{V}^{1}\left(Z^{\prime}\right)$ then choose $\tilde{w}$ extending $w$ as in Proposition 6. Since $Z \in \operatorname{Int}_{1}\left(H^{\infty}(\mathbb{B})\right)$, there exists $f \in H^{\infty}(\mathbb{B})$ such that $f_{\mid Z}=\tilde{w}$ and so $f_{\mid Z^{\prime}}=w$. 
Proof: Remark that we can see a sequence $w \in \mathcal{V}^{1}(Z)$ as a sequence of $\ell^{\infty}(\mathbb{C})$ satisfying Lipschitz conditions in the pseudohyperbolic metric. So, from this point of view, Proposition 6 is an extension problem for Lipschitz functions. We can find theorems about this type of problems in $[\mathbf{H i r} 80]$ and $[\mathbf{M c S 3 4}]$. More precisely, assume that $w$ is a real sequence of values over $Z^{\prime}$ satisfying Lipschitz conditions. Then, the proof of Theorem 1 of [Hir80] shows that

$$
\tilde{w}_{j}=\inf _{q \in I^{\prime}}\left\{w_{q}+M \rho\left(z_{q}, z_{j}\right)\right\}
$$

where $M=\|w\|_{\mathcal{V}^{1}(Z)}$, is a Lipschitz extension of $w$ and $\|\tilde{w}\|_{\mathcal{V}^{1}(Z)} \leq$ $\|w\|_{\mathcal{V}^{1}\left(Z^{\prime}\right)}$.

When $w_{q} \in \mathbb{C}$ set $w_{q}=u_{q}+i u_{q}^{\prime}$ where $u_{q}$ and $u_{q}^{\prime}$ are respectively the real part and imaginary part of $w_{q}$. Notice that $\left(u_{q}\right)_{q \in I^{\prime}}$ and $\left(u_{q}^{\prime}\right)_{q \in I^{\prime}}$

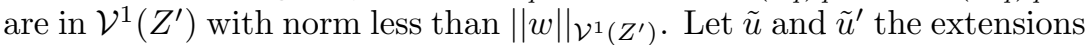
of $u$ and $u^{\prime}$ and define $\tilde{w}=\tilde{u}+i \tilde{u}^{\prime}$. Clearly $\tilde{w}$ extends $w$ and $\|\tilde{w}\|_{\mathcal{V}^{1}(Z)} \leq$ $\sqrt{2}\|w\|_{\mathcal{V}^{1}\left(Z^{\prime}\right)}$.

We will repeatedly use the following lemma where $\partial_{n}$ and $\partial_{t}$ denote partial derivatives in the normal and tangential direction.

Lemma 7. Let $f \in H^{\infty}(\mathbb{B})$ and $A<1 / \sqrt{2}$ then for all multi-index $\alpha=$ $\left(\alpha_{1}, \alpha_{2}\right)$ and using the notation $\xi^{\alpha}=\xi_{1}^{\alpha_{1}} \xi_{2}^{\alpha_{2}}$ for $\xi \in \mathbb{B}$, we have

(i) For all $a, b \in \mathbb{B}$ such that $\rho(a, b)<A$ we have

$$
\frac{1}{\alpha !}\left|\partial_{n}^{\alpha_{1}} \partial_{t}^{\alpha_{2}} f(a)(b-a)^{\alpha}\right| \leq\|f\|\left(\frac{\rho(a, b)}{A}\right)^{|\alpha|} .
$$

(ii) Let $q \in \mathbb{N}, a, b \in \mathbb{B}$ such that $\rho(a, b) \leq \frac{A}{2}$ and $S_{q} f(a)(b-a)$ be the remainder of order $q$ of the Taylor series of $f$ :

$$
S_{q} f(a)(b-a)=\sum_{|\alpha| \geq q} \frac{\partial_{n}^{\alpha_{1}} \partial_{t}^{\alpha_{2}} f(a)}{\alpha !}(b-a)^{\alpha} .
$$

Then there exists $C=C(q, A)$, such that

$$
\left|S_{q} f(a)(b-a)\right| \leq C\|f\| \rho^{q}(a, b) .
$$

Proof: (i) Let $\Delta_{H}(a, A)$ the pseudohyperbolic polydisk defined by

$$
\begin{aligned}
\Delta_{H}(a, A)=\left\{z=\lambda n_{a}+\beta t_{a}\right. \text { s.t. } & |\lambda-| a|| \\
& \left.\leq A\left(1-|a|^{2}\right) \text { and }|\beta| \leq A\left(1-|a|^{2}\right)^{1 / 2}\right\},
\end{aligned}
$$

where $n_{a}$ and $t_{a}$ respectively stand for the normalized vectors in the complex normal and tangential directions of $a$. The fact that $A \sqrt{2}<1$ implies that $\Delta_{H}(a, A) \subset \mathbb{B}$, and $\rho(a, b)<A$ implies $b \in \Delta_{H}(a, A)$. 
Applying the Cauchy formula on this polydisk we obtain using the notation $\alpha+1=\left(\alpha_{1}+1, \alpha_{2}+1\right)$ :

$$
\begin{aligned}
\left|\frac{\partial_{n}^{\alpha_{1}} \partial_{t}^{\alpha_{2}} f(a)}{\alpha !}(b-a)^{\alpha}\right| & =\left|\frac{1}{(2 \pi)^{2}} \int_{\partial_{0} \Delta_{H}(a, A)} \frac{f(\xi)}{(\xi-a)^{\alpha+1}} d \xi\right|\left|(b-a)^{\alpha}\right|, \\
& \leq\|f\| \frac{1}{A^{|\alpha|}\left(1-|a|^{2}\right)^{\alpha_{1}+\frac{\alpha_{2}}{2}}} \rho^{|\alpha|}(a, b)\left(1-|a|^{2}\right)^{\alpha_{1}+\frac{\alpha_{2}}{2}}, \\
& \leq\|f\|\left(\frac{\rho(a, b)}{A}\right)^{|\alpha|},
\end{aligned}
$$

where $\partial_{0} \Delta_{H}(a, A)$ is the distinguished border of $\Delta_{H}(a, A)$.

(ii) Applying (i) we have

$$
\begin{aligned}
\left|S_{q} f(a)(b-a)\right| & \leq\|f\| \sum_{|\alpha| \geq q}\left(\frac{\rho(a, b)}{A}\right)^{|\alpha|} \\
& \leq\|f\|\left(\frac{\rho(a, b)}{A}\right)^{q} \sum_{|\alpha| \geq q}\left(\frac{\rho(a, b)}{A}\right)^{|\alpha|-q}, \\
& \leq\|f\|\left(\frac{\rho(a, b)}{A}\right)^{q}(q+1)\left(\sum_{k=0}^{\infty}\left(\frac{\rho(a, b)}{A}\right)^{k}\right)^{2} \\
& \leq \frac{4(q+1)\|f\|}{A^{q}} \rho^{q}(a, b) .
\end{aligned}
$$

For the next to last inequality we use the fact that for $u<1$ and $S=$ $\sum_{k=0}^{\infty} u^{k}$, we have

$$
\begin{aligned}
\sum_{|\alpha| \geq q} u^{|\alpha|-q} & =\sum_{\alpha_{1}=0}^{q-1} \sum_{\alpha_{2}=q-\alpha_{1}}^{\infty} u^{\alpha_{1}-q} u^{\alpha_{2}}+\sum_{\alpha_{1}=q}^{\infty} \sum_{\alpha_{2}=0}^{\infty} u^{\alpha_{1}+\alpha_{2}-q} \\
& =\sum_{\alpha_{1}=0}^{q-1} u^{\alpha_{1}-q}\left(\frac{u^{q-\alpha_{1}}}{1-u}\right)+S^{2}=q S+S^{2} \leq(q+1) S^{2} .
\end{aligned}
$$




\section{Necessary condition in Theorem 2}

The key argument in this part is that if $f \in H^{\infty}(\mathbb{B})$ and $f$ vanishes at 3 points which define a coordinate system then the gradient of $f$ will be small on a ball containing these 3 points. Using this property for an interpolating function for a particular sequence of values we carry out a proof by contradiction.

Remark 8. We often use the following fact: Let $a \in \mathbb{D}$ and $f: \mathbb{D} \rightarrow \mathbb{D}$ be an analytic function satisfying $f(0)=f(a)=0$ then we have the estimate $\left|f^{\prime}(0)\right| \leq|a|$. Indeed, we have

$$
f(z)=z \cdot \frac{z-a}{1-z \bar{a}} g(z)
$$

where $g$ is holomorphic in $\mathbb{D}$ and $|g| \leq 1$ by the maximum principle. We have then $\left|f^{\prime}(0)\right|=|a . g(0)| \leq|a|$.

Notation. For $f$ analytic in $\mathbb{B}$ we define $\nabla f:=\left(\begin{array}{c}\frac{\partial f}{\partial z_{1}} \\ \frac{\partial f}{\partial z_{2}}\end{array}\right)$ the gradient of $f$.

Lemma 9. There exists $C>0$ such that for all $h<1 / 2, a, b \in B(0, h)$, and $f \in H^{\infty}(\mathbb{B})$ satisfying $f(0)=f(a)=f(b)=0$ we have for $\mathcal{A}(a, b) \neq$ 0 ,

$$
\|\nabla f(z)\| \leq \frac{C\|f\| h}{\sin \mathcal{A}(a, b)} \quad \text { uniformly for } z \in B(0, h) .
$$

Proof: First we prove this estimate for $z=0$. We can assume (with a rotation) that $b=\left(b_{1}, 0\right)$. Set $\theta=\mathcal{A}(a, b),\left(\right.$ so $\cos \theta=\left|a_{1}\right| /|a|$ and $\left.|\sin \theta|=\left|a_{2}\right| /|a|\right)$. By Remark 8

$$
\left|\frac{\partial f}{\partial z_{1}}(0)\right| \leq\|f\| \cdot|b| \leq\|f\| h .
$$

Let $g(t)=f\left(t \frac{a}{|a|}\right), g \in H^{\infty}(\mathbb{B}),\|g\| \leq\|f\|$ and $g(|a|)=g(0)=0$, so we have again by Remark $8\left|g^{\prime}(0)\right| \leq|| f|||a|$ and

$$
g^{\prime}(0)=\left\langle\nabla f(0), \frac{\bar{a}}{|a|}\right\rangle=\frac{\partial f}{\partial z_{1}}(0) \frac{a_{1}}{|a|}+\frac{\partial f}{\partial z_{2}}(0) \frac{a_{2}}{|a|},
$$

so

$$
\left|\frac{\partial f}{\partial z_{2}}(0)\right| \frac{\left|a_{2}\right|}{|a|} \leq \| f|| h+\left|\frac{\partial f}{\partial z_{1}}(0)\right| \frac{\left|a_{1}\right|}{|a|} \leq 2|| f|| h,
$$

which implies $\left|\frac{\partial f}{\partial z_{2}}(0)\right| \leq \frac{2 \| f|| h}{\sin \theta}$. 
To extend this estimate to $z \in B(0, h)$ recall the invariant Schwarz lemma (see [Rud80, p. 161]): For $f \in H^{\infty}(B(0, r))$, we have for $a, b \in$ $B(0, r)$

$$
|f(a)-f(b)| \leq 2\|f\| \rho\left(\frac{a}{r}, \frac{b}{r}\right) .
$$

Applying this to the function $g(z)=\frac{\partial f}{\partial z_{2}}(z)$ (for all $r<1$ we have $g \in H^{\infty}(B(0, r))$ and $\left.\|g\|_{H^{\infty}(B(0, r))} \leq \frac{\|f\|}{1-r^{2}}\right)$ we get

$$
|g(0)-g(z)| \leq \frac{2|| f||}{r\left(1-r^{2}\right)}|z|, \quad z \in B(0, h),
$$

and so for $z \in B(0, h)$ we have

$$
\left|\frac{\partial f}{\partial z_{2}}(z)\right| \leq \frac{C\|f\| h}{\sin \theta},
$$

the same method works to estimate $\left|\frac{\partial f}{\partial z_{1}}(z)\right|$, hence we get the result.

Lemma 10. Let $Z \in \operatorname{Int}_{1}\left(H^{\infty}\right)$ such that $Z=\left\{a_{k}^{(1)}\right\} \cup\left\{a_{k}^{(2)}\right\} \cup\left\{a_{k}^{(3)}\right\}$ where $\left(a_{k}^{(i)}\right)_{k \geq 0}$ are sequences of points in $\mathbb{B}$ satisfying $a_{k}^{(2)}, a_{k}^{(3)} \in$ $K\left(a_{k}^{(1)}, \gamma_{k}\right)$ with $\gamma_{k} \rightarrow 0$. There exist $\theta>0, N>0$ such that for any $k \geq N$ there is a permutation $\sigma \in \mathcal{S}_{3}$ such that $\mathcal{A}\left(\phi\left(a_{k}^{\sigma(2)}\right), \phi\left(a_{k}^{\sigma(3)}\right)\right) \geq \theta$ where $\phi=\phi_{a_{k}^{\sigma(1)}}$ is the automorphism exchanging 0 and $a_{k}^{\sigma(1)}$.

Proof: If it were not the case, we could find a subsequence $Z$ such that for all $\sigma \in \mathcal{S}_{3}$ we would have

$$
\mathcal{A}\left(\phi\left(a_{k}^{\sigma(2)}\right), \phi\left(a_{k}^{\sigma(3)}\right)\right) \leq \theta_{k} \quad \text { with } \theta_{k} \rightarrow 0,
$$

and

$$
\rho\left(a_{k}^{(i)}, a_{m}^{(j)}\right) \geq 2 \sup _{q \geq 0} \theta_{q} \quad \text { for any } k \neq m .
$$

Reordering $a_{k}^{(2)}, a_{k}^{(3)}$ if needed, we may assume $\rho\left(a_{k}^{(3)}, a_{k}^{(2)}\right) \leq \rho\left(a_{k}^{(1)}, a_{k}^{(3)}\right)$.

Define a sequence of values $w^{(m)}:=\left(\delta_{z_{k}}^{a_{m}^{(3)}}\right)_{k \geq 0}$. An easy calculation shows that

$$
\left\|w^{(m)}\right\|_{\mathcal{V}^{1}(Z)} \lesssim \frac{1}{\rho\left(a_{m}^{(2)}, a_{m}^{(3)}\right)}
$$

so using the fact that $Z$ is an interpolating sequence of order 1 (Proposition 6$)$, we can find $f_{m} \in H^{\infty}(\mathbb{B})$ such that $R_{Z}\left(f_{m}\right)=w^{(m)}$ and

$$
\left\|f_{m}\right\| \leq M\left\|w^{(m)}\right\| \lesssim \frac{1}{\rho\left(a_{m}^{(2)}, a_{m}^{(3)}\right)} .
$$


Let $\phi_{m}$ be the automorphism of $\mathbb{B}$ such that $\phi_{m}\left(a_{m}^{(1)}\right)=0, \phi_{m}\left(a_{m}^{(2)}\right) \in$ $\mathbb{C} \times\{0\}$ and define $g_{m}=f_{m} \circ \phi_{m}$ and $b_{m}^{(i)}=\phi_{m}\left(a_{m}^{(i)}\right), i=1,2,3$. Let $b^{i}$ and $g$ to denote respectively $b_{m}^{(i)}$ and $g_{m}$. The function $g$ satisfies

$$
\left\{\begin{array}{l}
\|g\| \lesssim \frac{1}{\rho\left(b^{(3)}, b^{(2)}\right)}, \quad \text { since } \rho \text { is invariant under automorphisms, } \\
g(0)=0, g\left(b^{(2)}\right)=0, g\left(b^{(3)}\right)=1 .
\end{array}\right.
$$

Then by Remark 8 we have

$$
\left|\frac{\partial g}{\partial z_{1}}\left(b^{(2)}\right)\right| \leq\|g\| \gamma_{m} \lesssim \frac{\gamma_{m}}{\rho\left(b^{(3)}, b^{(2)}\right)} .
$$

The Taylor expansion of $g$ in $b^{(2)}$ gives

(1) $g\left(b^{(3)}\right)=g\left(b^{(2)}\right)+\left\langle\nabla g\left(b^{(2)}\right), \overline{b^{(3)}-b^{(2)}}\right\rangle+S_{2} g\left(b^{(2)}\right)\left(b^{(3)}-b^{(2)}\right)$,

so

(2) $\quad 1=\frac{\partial g}{\partial z_{1}}\left(b^{(2)}\right) \cdot\left(b_{1}^{(3)}-b_{1}^{(2)}\right)$

$$
+\frac{\partial g}{\partial z_{2}}\left(b^{(2)}\right) \cdot\left(b_{2}^{(3)}-b_{2}^{(2)}\right)+S_{2} g\left(b^{(2)}\right)\left(b^{(3)}-b^{(2)}\right),
$$

(where subscripts now denote coordinates in $\mathbb{C}^{2}$ ), and

$$
\begin{aligned}
& \left|b_{2}^{(3)}-b_{2}^{(2)}\right| \leq \sin \theta_{m}\left|b^{(3)}-b^{(2)}\right|, \\
& \left|b_{1}^{(3)}-b_{1}^{(2)}\right| \leq\left|b^{(3)}-b^{(2)}\right| \simeq \rho\left(b^{(3)}, b^{(2)}\right), \\
& \left|S_{2} g\left(b^{(2)}\right)\left(b^{(3)}-b^{(2)}\right)\right| \lesssim \rho\left(b^{(2)}, b^{(3)}\right) \quad \text { by Lemma } 7, \\
& \left|\frac{\partial g}{\partial z_{2}}\left(b^{(2)}\right)\right| \leq \frac{|| g||}{1-\gamma_{m}} \lesssim \frac{1}{\rho\left(b^{(2)}, b^{(3)}\right)} .
\end{aligned}
$$

Carrying these estimates into (2), we have for all $m$

$$
1 \lesssim \gamma_{m}+\sin \theta_{m}+\rho\left(b_{m}^{(2)}, b_{m}^{(3)}\right)
$$

Letting $m$ tend to infinity, we get a contradiction, which proves the lemma.

Lemma 11. If $Z$ is an interpolating sequence of order $1, Z$ is a union of at most 3 separated sequences. 
Proof: By contradiction, passing if needed to a subsequence of $Z$ (see Section 2, Proposition 6), we may assume that $Z=\cup_{i=1}^{4}\left\{a_{k}^{(i)}\right\}, a_{k}^{(2)}, a_{k}^{(3)}$, $a_{k}^{(4)} \in K\left(a_{k}^{(1)}, \gamma_{k}\right)$ with $\gamma_{k} \rightarrow 0$ and $\rho\left(a_{k}^{(1)}, a_{q}^{(1)}\right) \geq \frac{1}{2}$ for $k \neq q$. By Lemma 10 and Proposition 6 we can assume furthermore, denoting $\phi=$ $\phi_{a_{k}^{(1)}}$ that $\mathcal{A}\left(\phi\left(a_{k}^{(2)}\right), \phi\left(a_{k}^{(3)}\right)\right) \geq \theta>0$. Let us define

$$
w^{(m)}=\left(w_{k}^{(m)}\right)_{k \in \mathbb{N}}=\left(\delta_{z_{k}}^{a_{m}^{(4)}}\right)_{k \in \mathbb{N}}
$$

and $a_{m}^{(5)}$ be a first neighbour of $a_{m}^{(4)}$ :

$$
\rho\left(a_{m}^{(4)}, a_{m}^{(5)}\right)=\inf \left\{\rho\left(a_{m}^{(4)}, z_{q}\right), z_{q} \in Z \backslash\left\{a_{m}^{(4)}\right\}\right\} .
$$

We have $\left\|w^{(m)}\right\| \lesssim \frac{1}{\rho\left(a_{m}^{(4)}, a_{m}^{(5)}\right)}$. By the interpolation property of $Z$, there exists $f_{m}$ such that $R_{Z}\left(f_{m}\right)=w^{(m)}$ and $\left\|f_{m}\right\| \lesssim \frac{1}{\rho\left(a_{m}^{(4)}, a_{m}^{(5)}\right)}$. Let $g:=g_{m}=f_{m} \circ \phi_{a_{m}^{(1)}}$ and $b^{(i)}:=b_{m}^{(i)}=\phi_{a_{m}^{(1)}}\left(a_{m}^{(i)}\right), i=1, \ldots, 5$. The function $g$ satisfies

$$
\|g\| \lesssim \frac{1}{\rho\left(b^{(4)}, b^{(5)}\right)}, \quad g(0)=g\left(b^{(2)}\right)=g\left(b^{(3)}\right)=0,
$$

so by Lemma 9

$$
\|\nabla g\| \lesssim \frac{\gamma_{m}}{\rho\left(b^{(4)}, b^{(5)}\right)} \quad \text { on } B\left(0, \gamma_{m}\right)
$$

But $g\left(b^{(4)}\right)=1$ and $g\left(b^{(5)}\right)=0$ so by the Taylor expansion of $g$ (as in (1)) we have

$$
\left|\nabla_{\left(b^{(4)}-b^{(5)}\right)} g\left(b^{(4)}\right)\right| \geq \frac{C}{\rho\left(b^{(4)}, b^{(5)}\right)},
$$

where $\nabla_{b^{(4)}-b^{(5)}} g$ denotes the derivatives of $g$ is the direction $b^{(4)}-b^{(5)}$. Hence we have a contradiction with (3).

Turn to the proof of the necessary condition in Theorem 2: Let $Z \in$ $\operatorname{Int}_{1}\left(H^{\infty}\right)$, Lemma 11 implies that $Z$ is a union of at most 3 separated sequences, and by Proposition 6 we deduce that $Z$ is a union of 3 free interpolating sequences since separated sequences of order 1 are free interpolating sequences. Moreover Lemma 10 allows us to conclude the proof of the necessary condition of Theorem 2 . 


\section{Sufficient condition}

We will show the sufficient condition by successive approximation. First we show that a separated union of interpolating sequences of order 1 remains an interpolating sequence of order 1 . Besides, if $Z$ is a sequence satisfying the sufficient condition of Theorem 2 we will separate it into 3 subsequences $B^{1}, B^{2}, B^{3}$ with $\rho\left(B^{i}, B^{j}\right) \geq c>0$ for which it is easy to show that each one is an interpolating sequence of order 1. More precisely, each $B^{i}$ will be made up of packets of at most 3 points and packets are separated. So, on each $B^{i}$, we will interpolate the correct value at one point in each packet and the best possible gradient in the direction of other points of the packet. The error will be small, and applying again recursively this approximate interpolation scheme to interpolate this error we get the correct interpolating function as a series.

\subsection{Decomposition of a union of 3 free interpolating sequences.}

Proposition 12. Let $Z$ be a disjoint union of 3 free interpolating sequences: $Z=A^{1} \cup A^{2} \cup A^{3}$; set $\eta$ the smallest separation constant of $A^{1}$, $A^{2}, A^{3}$ and $\delta<\eta$. There exist 3 disjoint subsequences of $Z, B^{1}, B^{2}, B^{3}$ such that

(i) $Z=B^{1} \cup B^{2} \cup B^{3}$.

(ii) For $i \neq j, \rho\left(B^{i}, B^{j}\right) \geq \delta / 8$.

(iii) There exists a family of packets of points of $Z,\left(G_{k}^{i}\right)_{k \in \mathbb{N}, 1 \leq i \leq 3}$ such that $\# G_{k}^{i} \leq i$, and $\forall i, j, m \neq k \rho\left(G_{k}^{i}, G_{m}^{j}\right) \geq \delta / 8, B^{i}=\cup_{k} G_{k}^{i}$ and the pseudohyperbolic diameter of $\left(G_{k}^{i}\right)$ is less than $\delta / 4$.

(iv) $A^{3} \subset B^{3}, A^{3} \cup A^{2} \subset B^{3} \cup B^{2}$.

The sequence $B^{3}$ is made up of $A^{3}$ and the points of $A^{1}$ and $A^{2}$ which are nearby. The sequence $B^{2}$ is define as the remainder of $A^{2}$ and the remaining points of $A^{1}$ which are near $A^{2}$. Then $B^{1}$ is what remains of $A^{1}$.

Definition 13. We will call the above decomposition the decomposition in packets of $Z$.

Remark 14. We deduce from the necessary condition of Theorem 2 that all interpolating sequences of order 1 admit a decomposition in packets.

Proof of Proposition 12: For $a_{k}^{(3)} \in A^{3}$, let $E_{k}=K\left(a_{k}^{(3)}, \delta / 8\right) \cap Z$ and set $G_{k}^{3}=Z \cap \cup_{a \in E_{k}} K(a, \delta / 8)$, that is $G_{k}^{3}$ consists of all the points $z \in$ $Z$ such that either $\rho\left(z, a_{k}^{(3)}\right)<\delta / 8$, or there exists $w \in Z$ such that $\rho\left(a_{k}^{(3)}, w\right)<\delta / 8$ and $\rho(z, w) \leq \delta / 8$. Set $B^{3}=\cup_{k \in \mathbb{N}} G_{k}^{3}$. Remark that 
$G_{k}^{3} \subset K\left(a_{k}^{(3)}, \delta / 4\right)$ so as $A^{3}$ is separated we have, for $m \neq k, \rho\left(G_{k}^{3}, G_{m}^{3}\right) \geq$ $\delta / 2$. We can also deduce that $\# G_{k}^{3} \leq 3$ because the 3 sequences making $Z$ are $\delta$-separated and $\operatorname{diam}\left(G_{k}^{3}\right) \leq \delta / 4$, so $G_{k}^{3}$ contains at most one point from each $A^{i}, i=1,2,3$.

We have $\rho\left(B^{3}, Z \backslash B^{3}\right) \geq \delta / 8$, indeed let $v \in Z \backslash B^{3}$ so $v \in A^{1} \cup A^{2}$, suppose $v \in A^{2}$ (the proof is similar for $v \in A^{1}$ ) so $v=a_{k}^{(2)}$ for some $k$. We have

- $\rho\left(A^{3}, a_{k}^{(2)}\right) \geq \delta / 8$ else $a_{k}^{(2)} \in B^{3}$ by construction of $B^{3}$.

- $\rho\left(A^{2} \backslash\left\{a_{k}^{(2)}\right\},\left\{a_{k}^{(2)}\right\}\right) \geq \delta$ because $A^{2}$ is $\delta$-separated.

- $\rho\left(a_{k}^{(2)}, A^{1} \cap B^{3}\right) \geq \delta / 8$ otherwise there exists $a_{p}^{(1)} \in B^{3}$ with $\rho\left(a_{p}^{(1)}, a_{k}^{(2)}\right)<\delta / 8$, as $a_{p}^{(1)} \in B^{3}$ there are 2 possible cases reaching a contradiction:

1) There exists $a_{q}^{(3)} \in A^{3}$ such that $\rho\left(a_{q}^{(3)}, a_{p}^{(1)}\right)<\delta / 8$ and so by construction of $B^{3}, a_{k}^{(2)} \in B^{3}$ which is a contradiction.

2) There exists $a_{q}^{(3)} \in A^{3}$, and $a_{j}^{(2)} \in A^{2} \cap B^{3}$ such that $\rho\left(a_{q}^{(3)}, a_{j}^{(2)}\right)<\delta / 8$ and $\rho\left(a_{p}^{(1)}, a_{j}^{(2)}\right)<\delta / 8$ so $\rho\left(a_{k}^{(2)}, a_{j}^{(2)}\right)<\delta / 4$ and since $A^{2}$ is $\delta$-separated and $a_{k}^{(2)} \notin B^{3}$, it is a contradiction.

For the construction of $B^{1}$ and $B^{2}$, we apply the same process to the sequence $Z \backslash B^{3}$. We set for $a_{k}^{(2)} \in A^{2} \backslash B^{3}, F_{k}=K\left(a_{k}^{(2)}, \delta / 8\right) \cap\left(Z \backslash B^{3}\right)$ and we define $G_{k}^{2}=F_{k}, B^{2}=\cup_{k \in \mathbb{N}} G_{k}^{2}$ and $B^{1}=Z \backslash\left(B^{3} \cup B^{2}\right)$. Since $\rho\left(B^{3}, Z \backslash B^{3}\right) \geq \delta / 8$ we have $\rho\left(B^{3}, B^{j}\right) \geq \delta / 8$ for $j=1,2$. By the same method as above we show that $B^{2}$ and $B^{3}$ satisfy the desired properties.

\subsection{Separated union of separated sequence.}

Drury in [Dru72], has shown (cf. also N. T. Varopoulos [Var71]) that a separate union of 2 free interpolating sequences is again a free interpolating sequence. Modifying the proof of [Var71] we will show that this is also true for interpolating sequences of order 1.

Proposition 15. Let $Z$ and $Z^{\prime}$ be interpolating sequences of order 1 for $H^{\infty}(\mathbb{B})$ such that $\rho\left(Z, Z^{\prime}\right)>\gamma>0$. Then $Z \cup Z^{\prime}$ remains an interpolating sequence of order 1 .

We will use the discrete Fourier transform. Let $\left(f_{j}\right)_{j \in\{0, \ldots, N\}} \in \mathbb{C}^{N+1}$, and $\lambda=e^{2 i \pi /(N+1)}$ be a $(N+1)$ th root of unity, we define

$$
\begin{aligned}
\mathcal{F}: \mathbb{C}^{N+1} & \longrightarrow \mathbb{C}^{N+1} \\
\left(f_{j}\right) & \longrightarrow\left(\hat{f}_{k}\right)
\end{aligned} \quad \text { with } \quad \hat{f}_{k}=\frac{1}{N+1} \sum_{j=0}^{N} f_{j} \lambda^{-j k}
$$


Then we have the Fourier inversion and Parseval formulas:

For all $p \in\{0, \ldots, N\} \quad f_{p}=\sum_{j=0}^{N} \hat{f}_{j} \lambda^{j p}, \quad \sum_{k=0}^{N}\left|\hat{f}_{k}\right|^{2}=\frac{1}{N+1} \sum_{j=0}^{N}\left|f_{j}\right|^{2}$.

The proof of Proposition 15 is based on the following lemma:

Lemma 16. Let $Z$ be an interpolating sequence of order 1 for $H^{\infty}$. Assume that $Z$ admits a decomposition in packets (cf. Definition 13) with $\mu$ the minimal distance between 2 packets.

Let $E$ be a sequence of $\mathbb{B}$ satisfying $\rho(E, Z) \geq \gamma>0$ where $\gamma$ is some constant and assume that there exists $L>0$ such that for any packet $G$ of $Z$, there exists $f_{G} \in H^{\infty}(\mathbb{B})$ with $\left\|f_{G}\right\| \leq L, f_{G} \equiv 1$ on $G$, and $f_{G} \equiv 0$ on $E$. Then there exists $F \in H^{\infty}(\mathbb{B})$ such $F \equiv 1$ on $Z$ and $F \equiv 0$ on $E$. Moreover $\|F\| \lesssim\left(\frac{M_{1}(Z)}{\mu}\right)^{2} L$.

Notice that we can deduce $\rho(E, Z) \geq \gamma$ from the existence of the constant $L$ and from the family of functions $f_{G}$. Indeed the invariant Schwarz Lemma shows

$$
\rho\left(\frac{f_{G}(g)}{\|f\|}, \frac{f_{G}(e)}{\|f\|}\right) \leq \rho(g, e) \text { for all } g \in G \text { and } e \in E,
$$

and this equation is true for all packets $G$ so we deduce $\rho(E, Z) \geq 1 / L$. Proof: Set $G_{0}, \ldots, G_{N}$ the first $N+1$ packets in the decomposition of $Z$. As $Z$ is an interpolating sequence of order 1 there exist $\beta_{j} \in H^{\infty}(\mathbb{B})$ for $j=0, \ldots, N$ such that

$$
\left\{\begin{array}{l}
\beta_{j \mid G_{k}}=\lambda^{j k} \\
\left\|\beta_{j}\right\| \lesssim \frac{M_{1}(Z)}{\mu}
\end{array} \quad \text { for all } k \in\{0, \ldots, N\},\right.
$$

where the second inequality is obtained using the fact that $\left\|\lambda^{j k}\right\|_{\mathcal{V}^{1}(Z)} \leq$ $\frac{2}{\mu} \lesssim 1 / \mu$ (we interpolate constant values of modulus less than 1 on each packet and the distance between 2 packets is more than $\mu$ ).

For $z \in \mathbb{B},\left(\beta_{j}(z)\right)_{j=0, \ldots, N}$ is a sequence of $N+1$ complex numbers. We extend it over $\mathbb{Z}$ by periodicity: for all $p \in\{0, \ldots, N\}$ and $k \in \mathbb{Z}$, $\beta_{p+k(N+1)}(z):=\beta_{p}(z)$.

Let

and define

$$
\delta_{j}(z)=\frac{1}{N+1} \sum_{k=0}^{N} \beta_{j-k}(z) \beta_{k}(z),
$$

$$
F_{N}(z)=\sum_{j=0}^{N} \hat{f}_{G_{j}}(z) \delta_{j}(z)
$$


$F_{N}$ satisfies

(i) $\quad F_{N \mid G_{j}} \equiv 1$ for all $j=0, \ldots, N$,

(ii) $\quad F_{N \mid E} \equiv 0$,

(iii) $\quad F_{N} \in H^{\infty}(\mathbb{B})$ and $\left\|F_{N}\right\| \lesssim L\left(\frac{M_{1}(Z)}{\mu}\right)^{2}$.

Indeed, for (i) Let $z \in G_{q}$ then

$$
\begin{aligned}
\delta_{j}(z) & =\frac{1}{N+1} \sum_{k=0}^{N} \beta_{j-k}(z) \beta_{k}(z) \\
& =\frac{1}{N+1} \sum_{k=0}^{N} \lambda^{(j-k) q} \lambda^{k q} \\
& =\frac{1}{N+1} \sum_{k=0}^{N} \lambda^{j q}=\lambda^{j q},
\end{aligned}
$$

so $F_{N}(z)=\sum_{j=0}^{N} \hat{f}_{G_{j}}(z) \lambda^{j q}=f_{G_{q}}(z)=1$.

(ii) $F_{N}$ is a linear combination of $\hat{f}_{G_{j}}(z)$ and each one is itself a linear combination of $f_{G_{k}}(z)$ and for $z \in E, f_{G_{k}}(z)=0$.

(iii) Note that a Fourier transform of a convolution $f \star g$ is the product of the Fourier transform of $f$ and $g$ so

$$
\left|\hat{\delta}_{k}(z)\right|=\left|\hat{\beta}_{k}(z)\right|^{2}
$$

We deduce that

$$
\begin{aligned}
\left|F_{N}(z)\right| & =\left|\sum_{j=0}^{N} \hat{f}_{G_{j}}(z) \sum_{k=0}^{N} \hat{\delta}_{k}(z) \lambda^{k j}\right|=\left|\sum_{k=0}^{N} f_{G_{k}}(z) \hat{\delta}_{k}(z)\right| \\
& \leq L \sum_{k=0}^{N}\left|\hat{\beta}_{k}(z)\right|^{2}=\frac{L}{N+1} \sum_{k=0}^{N}\left|\beta_{k}(z)\right|^{2} \lesssim L\left(\frac{M_{1}(Z)}{\mu}\right)^{2}
\end{aligned}
$$

We then conclude the proof of the lemma by a normal family argument.

Proof of Proposition 15: First we show that there exists $F \in H^{\infty}(\mathbb{B})$, $F \equiv 1$ on $Z$ and $F \equiv 0$ on $Z^{\prime}$. 
$Z$ and $Z^{\prime}$ are interpolating sequences of order 1 so by Theorem $2, Z$ and $Z^{\prime}$ are union of 3 free interpolating sequences for $H^{\infty}$. So these sequences admit a decomposition in packets (see Remark 14): $Z=A^{1} \cup$ $A^{2} \cup A^{3}, Z^{\prime}=B^{1} \cup B^{2} \cup B^{3}$. Let $\left(G_{i}\right)_{i \in I}$ be the packets of $Z$ and $\left(G^{\prime}{ }_{i}\right)_{i \in I^{\prime}}$ the packets of $Z^{\prime}$, set

$$
\mu=\min \left(\inf _{i \neq j \in I} \rho\left(G_{i}, G_{j}\right), \inf _{i \neq j \in I^{\prime}} \rho\left(G_{i}^{\prime}, G_{j}^{\prime}\right)\right) .
$$

For $z^{\prime} \in Z^{\prime}$ and $G$ a packet of $Z$, there exists $f_{G} \in H^{\infty}(\mathbb{B})$ such that $f_{G_{\mid G}} \equiv 1, f_{G}\left(z^{\prime}\right)=0$ and $\left\|f_{G}\right\| \leq 1+1 / \gamma^{3}$. Indeed, $\rho\left(G, z^{\prime}\right) \geq \gamma$ so, for all $a \in G$, there exists $f_{a} \in H^{\infty}(\mathbb{B})$ such that $\left\|f_{a}\right\| \leq 1 / \gamma, f_{a}(a)=0$, $f_{a}\left(z^{\prime}\right)=1\left(\right.$ take $\left.f_{a}=\frac{\left\langle\phi_{a}, \phi_{a}\left(z^{\prime}\right)\right\rangle}{\left\|\phi_{a}\left(z^{\prime}\right)\right\|}\right)$. We then define $f_{G}=1-\prod_{a \in G} f_{a}$ and as $\# G \leq 3$ we have $\left\|f_{G}\right\| \leq 1+1 / \gamma^{3}$.

Applying Lemma 16 with $E=\left\{z^{\prime}\right\}$, we can construct for all $z^{\prime}$ in $Z^{\prime}, F_{z^{\prime}} \in H^{\infty}(\mathbb{B})$ such that $F_{z^{\prime}} \equiv 1$ on $Z, F_{z^{\prime}}\left(z^{\prime}\right)=0$, and $\left\|F_{z^{\prime}}\right\| \lesssim$ $\left(\frac{M_{1}(Z)}{\mu}\right)^{2}\left(1+1 / \gamma^{3}\right)$.

For any packet $G$ of $Z^{\prime}$, we set $h_{G}=1-\prod_{b \in G} F_{b}$. Then $h_{G_{\mid G}} \equiv 1$, $h_{G_{\mid Z}} \equiv 0$, and

$$
\left\|h_{G}\right\| \lesssim 1+\left(1+1 / \gamma^{3}\right)^{3}\left(\frac{M_{1}(Z)}{\mu}\right)^{6} .
$$

Applying again Lemma 16 with $E=Z$, there exists $F \in H^{\infty}(\mathbb{B})$ such that $F_{\mid Z^{\prime}} \equiv 1, F_{\mid Z} \equiv 0$, and

$$
\begin{aligned}
\|F\| & \lesssim\left(\frac{M_{1}\left(Z^{\prime}\right)}{\mu}\right)^{2}\left(1+\left(1+1 / \gamma^{3}\right)^{3}\left(\frac{M_{1}(Z)}{\mu}\right)^{6}\right)^{2}, \\
& \lesssim\left(M_{1}\left(Z^{\prime}\right)\right)^{2} \frac{\left(M_{1}(Z)\right)^{12}}{\mu^{14} \gamma^{18}} .
\end{aligned}
$$

In the same way, we can construct $H \in H^{\infty}(\mathbb{B})$ such that $H_{\mid Z^{\prime}} \equiv 0$, $H_{\mid Z} \equiv 1$, and

$$
\|H\| \lesssim\left(M_{1}(Z)\right)^{2} \frac{\left(M_{1}\left(Z^{\prime}\right)\right)^{12}}{\mu^{14} \gamma^{18}} .
$$

Let us prove that $Z \cup Z^{\prime}$ is an interpolating sequence of order 1 for $H^{\infty}$. Let $W \in \mathcal{V}_{1}\left(Z \cup Z^{\prime}\right)$, we can split $W$ into 2 sequences $W_{Z} \in \mathcal{V}_{1}(Z)$ and $W_{Z^{\prime}} \in \mathcal{V}_{1}\left(Z^{\prime}\right)$ in a natural way. The sequences $Z$ and $Z^{\prime}$ being interpolating sequences of order 1 , there exist $g_{Z}$ and $g_{Z^{\prime}}$ interpolating respectively $W_{Z}$ on $Z$ and $W_{Z^{\prime}}$ on $Z^{\prime}$. Set $\psi=H g_{Z}+F g_{Z^{\prime}}$. The 
function $\psi$ clearly interpolates $W$ on $Z \cup Z^{\prime}$ and moreover

$$
\|\psi\| \lesssim \frac{\left(M_{1}\left(Z^{\prime}\right) M_{1}(Z)\right)^{3}}{\mu^{14} \gamma^{18}}\left(M_{1}^{12}\left(Z^{\prime}\right)+M_{1}^{12}(Z)\right)\|W\|_{\mathcal{V}_{1}\left(Z \cup Z^{\prime}\right)},
$$

$$
M_{1}\left(Z \cup Z^{\prime}\right) \lesssim \frac{\left(M_{1}\left(Z^{\prime}\right) M_{1}(Z)\right)^{3}}{\mu^{14} \gamma^{18}}\left(M_{1}^{12}\left(Z^{\prime}\right)+M_{1}^{12}(Z)\right)
$$

\subsection{Multiple interpolation.}

In this section, we prove Theorem 5. I would to thank E. Amar for helpful suggestions to simplify the proof of this theorem. Assume that $Z$ is a free interpolating sequence and recall the following result (see also [Gar81, p. 294]):

Theorem (A. Bernard [Ber71]). Let $Z$ be a free interpolating sequence and $M$ the constant of interpolation of $Z$. There exists a family of functions $\beta_{k} \in H^{\infty}(\mathbb{B}), k \in \mathbb{N}$ such that

$$
\begin{array}{ll}
\text { (i) } & \beta_{k}\left(z_{j}\right)=\delta_{k}^{j}, \\
\text { (ii) } & \sum\left|\beta_{k}(z)\right|^{2} \leq M^{2} \text { uniformly for } z \in \mathbb{B} .
\end{array}
$$

Let $\left(l_{k}^{(j)}\right) \in \mathcal{M}^{i}(Z)$ and let prove that there exists $F \in H^{\infty}(\mathbb{B})$ such that $D^{j}\left(F \circ \phi_{k}\right)(0)=l_{k}^{(j)}$ for all $k \in \mathbb{N}, j=0, \ldots, i$. We will prove the result by induction on $i$. For $i=0$ the result is obvious. Assuming the result for the step $i-1$, we will prove it for the step $i$. By the inductive hypothesis, there exists $g \in H^{\infty}(\mathbb{B})$ such that $D^{j}\left(g \circ \phi_{k}\right)(0)=l_{k}^{(j)}$ for all $k \in \mathbb{N}$ and $j \leq i-1$. To solve the problem we just have to find $f \in H^{\infty}(\mathbb{B})$ such that

(10) $\begin{cases}D^{j}\left(f \circ \phi_{z_{k}}\right)(0)=0 & \text { for all } j \leq i-1 \text { and } k \in \mathbb{N}, \\ D^{i}\left(f \circ \phi_{z_{k}}\right)(0)=l_{k}^{(i)}-D^{i}\left(g \circ \phi_{k}\right)(0) & \text { for all } k \in \mathbb{N} .\end{cases}$

Indeed, if such an $f$ exists then $F=f+g$ is a solution of our problem.

Let $u_{k}:=l_{k}^{(i)}-D^{i}\left(g \circ \phi_{k}\right)(0), u_{k} \in \mathcal{L}_{i}\left(\mathbb{C}^{2}\right)$ and set $\tilde{u}_{k}$ the unique homogeneous polynomial of degree $i$ such that $D^{i} \tilde{u}_{k}(0)=u_{k}$. By the Cauchy formula, it is easy to show that $\sup _{k \in \mathbb{N}}\left\||| D^{i}\left(g \circ \phi_{k}\right)(0)\right\| \|<\infty$ since all partial derivatives are bounded uniformly for $k \in \mathbb{N}$. We deduce that $\left(u_{k}\right)_{k \in \mathbb{N}} \in \mathcal{M}^{i}(Z)$. Let us define

$$
f(z)=\sum_{k=0}^{\infty} \beta_{k}^{1+i}(z) \tilde{u}_{k}\left(\phi_{k}(z)\right) .
$$


The function $f$ is a solution of (10). Indeed, the above series converges normally by (9). Since the power of $\beta_{k}$ in $f$ is $1+i$, each $D^{j}\left(\beta_{k}^{1+i} \circ \phi_{q}\right)(0)$ contains a factor $\beta_{k}\left(\phi_{q}(0)\right)=\beta_{k}\left(z_{q}\right)=0$ for all $j \leq i$ and $q \neq k$, so when we compute $D^{j}\left(f \circ \phi_{q}\right)$ using (11) we only retain the $q$-th term and so

$$
D^{j}\left(f \circ \phi_{q}\right)(0)=D^{j}\left[\left(\beta_{q}^{i+1} \circ \phi_{q}\right) \cdot \tilde{u}_{q}\right](0) .
$$

If $j<i$ we have $D^{j}\left(\tilde{u}_{q}\right)(0)=0$ so finally $f$ satisfies the first part of (10).

Turn to the second part: let $\alpha=\left(\alpha_{1}, \alpha_{2}\right)$ be a multi-index such that $|\alpha|=i$ and let us compute the partial derivatives of $f \circ \phi_{q}$ :

$$
\begin{aligned}
\partial_{\alpha}\left(f \circ \phi_{q}\right)(0):=\frac{\partial^{\alpha_{1}} \partial^{\alpha_{2}}}{\partial z_{1}^{\alpha_{1}} \partial z_{2}^{\alpha_{2}}}( & \left.f \circ \phi_{q}\right)(0) \\
& =\sum_{\gamma \leq \alpha}\left(\begin{array}{l}
\alpha \\
\gamma
\end{array}\right) \partial_{\alpha-\gamma}\left(\beta_{q}^{i+1} \circ \phi_{q}\right)(0) \partial_{\gamma} \tilde{u}_{q}(0) .
\end{aligned}
$$

In the last sum, all terms are zero except when $\gamma=\alpha$ since $\partial_{\gamma} \tilde{u}_{q}(0)=0$ for $\gamma<\alpha$. We deduce

$$
\partial_{\alpha}\left(f \circ \phi_{q}\right)(0)=\left(\beta_{q}^{i+1} \circ \phi_{q}\right)(0) \partial_{\alpha} \tilde{u}_{q}(0)=\partial_{\alpha} \tilde{u}_{q}(0),
$$

which implies $D^{i}\left(f \circ \phi_{q}\right)(0)=D^{i}\left(\tilde{u}_{q}\right)(0)=u_{q}$ which completes the proof of Theorem 5 .

\subsection{Another preliminary result.}

Proposition 17. Let $Z=\left\{z_{k}\right\}$ be an interpolating sequence for $H^{\infty}$, $\eta$ the separation constant of $Z, \theta>0$. There exists $\delta_{0}(\theta, \eta)$ such that for all $\delta \leq \delta_{0}$ and all sequences $Z^{1}=\left\{z_{k}^{(1)}\right\}$ and $Z^{2}=\left\{z_{k}^{(2)}\right\}$ satisfying $z_{k}^{(i)} \in K\left(z_{k}, \delta\right) \backslash\left\{z_{k}\right\}, i=1,2$ and $\mathcal{A}\left(\phi_{z_{k}}\left(z_{k}^{(1)}\right), \phi_{z_{k}}\left(z_{k}^{(2)}\right)\right) \geq \theta$, we can solve the following problem:

For any sequences of values $\left(v_{k}\right),\left(u_{k}^{(1)}\right),\left(u_{k}^{(2)}\right)$ such that

$$
M:=\sup _{\substack{k \in \mathbb{N} \\ i=1,2}} \frac{\left|u_{k}^{(i)}\right|}{\rho\left(z_{k}^{(i)}, z_{k}\right)}+\sup _{k \in \mathbb{N}}\left|v_{k}\right|<\infty
$$

there exists $g \in H^{\infty}(\mathbb{B})$ such that

and

$$
\left\{\begin{array}{l}
g\left(z_{k}\right)=v_{k} \\
D g\left(z_{k}\right)\left(z_{k}^{(1)}-z_{k}\right)=u_{k}^{(1)} \\
D g\left(z_{k}\right)\left(z_{k}^{(2)}-z_{k}\right)=u_{k}^{(2)}
\end{array}\right.
$$

$$
\|g\| \leq C(Z, \theta) M
$$

where $C(Z, \theta)$ is a constant depending only on $Z$ and $\theta$. 
Proof: Assume that $\delta$ is small enough (we will say how small later). Let $t_{k}^{(i)}=D \phi_{k}\left(z_{k}\right)\left(z_{k}^{(i)}-z_{k}\right)$, for $i=1,2$. Note that by Lemma 7 we have

$$
t_{k}^{(i)}=\phi_{k}\left(z_{k}^{(i)}\right)+\epsilon_{k}^{(i)} \text { for } i=1,2,
$$

with $\left|\epsilon_{k}^{(i)}\right| \leq C \rho^{2}\left(z_{k}, z_{k}^{(i)}\right)$ where $C$ is an absolute constant. Let $e_{k}^{(i)}=$ $\frac{t_{k}^{(i)}}{\left|t_{k}^{(i)}\right|}$. The vectors $\left\{e_{k}^{(1)}, e_{k}^{(2)}\right\}$ form a basis of $\mathbb{C}^{2}$ and there exists $\theta^{\prime}>0$ such that $\mathcal{A}\left(e_{k}^{(1)}, e_{k}^{(2)}\right) \geq \theta^{\prime}$. Indeed, using (12) we have

$$
\left|t_{k}^{(i)}\right| \geq\left|\phi_{k}\left(z_{k}^{(i)}\right)\right|-\left|\epsilon_{k}^{(i)}\right| \geq\left|\phi_{k}\left(z_{k}^{(i)}\right)\right|(1-C \delta),
$$

$\mathrm{SO}$

$$
\begin{aligned}
\cos & \mathcal{A}\left(e_{k}^{(1)}, e_{k}^{(2)}\right) \\
& =\frac{\left\langle t_{k}^{(1)}, t_{k}^{(2)}\right\rangle}{\left|t_{k}^{(1)}\right| \cdot\left|t_{k}^{(1)}\right|} \\
& \leq \frac{\left\langle\phi_{k}\left(z_{k}^{(1)}\right), \phi_{k}\left(z_{k}^{(2)}\right)\right\rangle+\left\langle\phi_{k}\left(z_{k}^{(1)}\right), \epsilon_{k}^{(2)}\right\rangle+\left\langle\epsilon_{k}^{(1)}, \phi_{k}\left(z_{k}^{(2)}\right)\right\rangle+\left\langle\epsilon_{k}^{(1)}, \epsilon_{k}^{(2)}\right\rangle}{\left|\phi_{k}\left(z_{k}^{(1)}\right)\right|\left|\phi_{k}\left(z_{k}^{(2)}\right)\right|(1-C \delta)^{2}} \\
& \leq \frac{\cos \theta}{(1-C \delta)^{2}}+\frac{3 C \delta}{(1-C \delta)^{2}}<1 \text { for } \delta \text { small enough. }
\end{aligned}
$$

Set $l_{k}$ a family of linear maps defined by

$$
\begin{aligned}
l_{k}: \mathbb{C}^{2} & \longrightarrow \mathbb{C}, \\
e_{k}^{(i)} & \longrightarrow v_{k}^{(i)}:=\frac{u_{k}^{(i)}}{\left|t_{k}^{(i)}\right|} .
\end{aligned}
$$

Claim 18. The family $\left\{l_{k}\right\}$ is bounded (that is $\sup _{k \in \mathbb{N}}||\left|l_{k}\right|||<\infty$ ).

Indeed, first we have $\left(v_{k}^{(i)}\right)_{k \in \mathbb{N}} \in \ell^{\infty}$ for $i=1,2$ :

$$
\left|v_{k}^{(i)}\right|=\frac{\left|u_{k}^{(i)}\right|}{\left|t_{k}^{(i)}\right|} \leq \frac{1}{1-C \rho\left(z_{k}, z_{k}^{(i)}\right)} \sup _{k \in \mathbb{N}}\left(\frac{\left|u_{k}^{(i)}\right|}{\rho\left(z_{k}, z_{k}^{(i)}\right)}\right)<\infty .
$$

By Gram Schmidt applied to the basis $\left\{e_{k}^{(1)}, e_{k}^{(2)}\right\}$, we obtain an orthonormal basis $\left\{f_{k}^{(1)}, f_{k}^{(2)}\right\}$ with

$$
\left\{\begin{array}{l}
f_{k}^{(1)}=e_{k}^{(1)}, \\
f_{k}^{(2)}=\frac{1}{\sin \theta^{\prime}}\left(e_{k}^{(2)}-\left\langle e_{k}^{(2)}, e_{k}^{(1)}\right\rangle e_{k}^{(1)}\right),
\end{array}\right.
$$


therefore the matrix of $l_{k}$ in the basis $\left\{f_{k}^{(1)}, f_{k}^{(2)}\right\}$ is

$$
\operatorname{Mat}\left(l_{k}\right)=\left(v_{k}^{(1)}, \frac{1}{\sin \theta^{\prime}}\left(v_{k}^{(2)}-\left\langle e_{k}^{(2)}, e_{k}^{(1)}\right\rangle v_{k}^{(1)}\right)\right) \text {. }
$$

As it is an orthonormal basis, we deduce the claim since all coefficients of the matrix are bounded. The proposition follows: by Theorem 5 there exists $g \in H^{\infty}(\mathbb{B})$ such that

$$
D\left(g \circ \phi_{k}\right)(0)=l_{k}
$$

so $D\left(g \circ \phi_{k}\right)(0) t_{k}^{(i)}=u_{k}^{(i)}$ which implies the result by the fact that

$D\left(g \circ \phi_{k}\right)(0) t_{k}^{(i)}=D g\left(z_{k}\right) D \phi_{k}(0) D \phi_{k}\left(z_{k}\right)\left(z_{k}^{(i)}-z_{k}\right)=D g\left(z_{k}\right)\left(z_{k}^{(i)}-z_{k}\right)$, since $D \phi_{k}(0)=\left[D \phi_{k}\left(z_{k}\right)\right]^{-1}$.

\subsection{Proof of the sufficient condition in Theorem 2.}

Let $Z=A^{1} \cup A^{2} \cup A^{3}$ be a sequence satisfying the sufficient condition in Theorem 2 and set $\eta$ the smallest constant of separation. Let $\delta<\eta / 8$ and let $B^{1} \cup B^{2} \cup B^{3}$ the decomposition in packets of $Z$ such that $A^{3} \subset B^{3}$, $A^{3} \cup A^{2} \subset B^{2}$ and such that the distance between 2 packets is greater than $\delta$, and the diameter of packets is less than $2 \delta$. By Lemma 15 it suffices to show that $B^{1}, B^{2}, B^{3}$ are interpolating sequences of order 1 . We will show it for $B^{3}$. The proof for $B^{2}$ and $B^{3}$ is similar but simpler since packets of $B^{1}$, and $B^{2}$ have cardinal less than 2 .

Denote $B:=B^{3}, G_{k}$ the packets of $B$. The sequence $B$ have packets with cardinal at most 3 and, if needed, we can add some points to the sequences $A^{1}$ and $A^{2}$ and consider by Proposition 6 that the cardinal of packets of $B$ is 3 . We write

$$
G_{k}=\left\{e_{k}^{1}, e_{k}^{2}, e_{k}^{3}\right\}
$$

such that $e_{k}^{1} \in A^{3}$, and $e_{k}^{2}, e_{k}^{3} \in A^{2} \cup A^{1}$. Let $w$ in $\mathcal{V}^{1}(B)$, we have to show:

(13) There exists $f \in H^{\infty}(\mathbb{B})$ such that for all $b_{k} \in B, f\left(b_{k}\right)=w_{k}$.

Set $v_{k}^{i}, i=1,2,3$ the values of $w$ induced by the decomposition of $B=$ $\cup G_{k}$ : if $G_{k} \cap B^{3}=\left\{b_{p_{1}}, b_{p_{2}}, b_{p_{3}}\right\}$ and $b_{p_{i}}=e_{k}^{i}$, we then define $v_{k}^{i}=w_{p_{i}}$. Problem (13) can be rewritten as:

(14) Find $f \in H^{\infty}(\mathbb{B})$ such that for all $k \in \mathbb{N}$,

$$
\text { and } i=1,2,3, f\left(e_{k}^{i}\right)=v_{k}^{i} \text {. }
$$


By Proposition 17, for $\delta<\delta_{0}(\theta, \eta)$ there exists $g_{0} \in H^{\infty}(\mathbb{B})$ such that

$$
\left\{\begin{array}{l}
g_{0}\left(e_{k}^{1}\right)=v_{k}^{1}, \\
D g_{0}\left(e_{k}^{1}\right)\left(e_{k}^{2}-e_{k}^{1}\right)=v_{k}^{2}-v_{k}^{1}, \\
D g_{0}\left(e_{k}^{1}\right)\left(e_{k}^{3}-e_{k}^{1}\right)=v_{k}^{3}-v_{k}^{1}
\end{array}\right.
$$

and $\left\|g_{0}\right\| \leq C\left(A^{3}, \theta\right)\|w\|_{\mathcal{V}^{1}(B)}$. Let us evaluate the error $w^{1}=w-g_{0 \mid B}$ which has been induced by interpolating in this sense: the properties of the decomposition of $Z$ in packets allow us to write

$$
\begin{aligned}
|| w^{1} \mid \mathcal{V}^{1}(B) \leq & \underbrace{\sup _{k}\left|v_{k}^{i}-g_{0}\left(e_{k}^{i}\right)\right|}_{I} \\
& +\underbrace{\sup _{k}\left|\frac{v_{k}^{i}-g_{0}\left(e_{k}^{i}\right)-\left(v_{p}^{j}-g_{0}\left(e_{p}^{j}\right)\right)}{\rho\left(e_{k}^{i}, e_{p}^{j}\right)}\right|}_{\substack{e_{k}^{i}, e_{p}^{j} \in B \\
e_{p}^{j} \in K\left(e_{k}^{i}, \delta\right)}} \\
& +\underbrace{\sup _{\substack{e_{k}^{i}, e_{p}^{j} \in B \\
\left(e_{p}^{j}, e_{k}^{i}\right) \geq \delta}}\left|\frac{v_{k}^{i}-g_{0}\left(e_{k}^{i}\right)-\left(v_{p}^{j}-g_{0}\left(e_{p}^{j}\right)\right)}{\rho\left(e_{k}^{i}, e_{p}^{j}\right)}\right|}_{I I I} .
\end{aligned}
$$

Estimation of $I$ : we have $\left|g_{0}\left(e_{k}^{i}\right)-v_{k}^{i}\right|=0$ for $i=1$, and for $i=2,3$ we have

$$
\begin{aligned}
\left|g_{0}\left(e_{k}^{i}\right)-v_{k}^{i}\right| & =\left|g_{0}\left(e_{k}^{1}\right)+D g_{0}\left(e_{k}^{1}\right)\left(e_{k}^{i}-e_{k}^{1}\right)+S_{2} g_{0}\left(e_{k}^{1}\right)\left(e_{k}^{i}-e_{k}^{1}\right)-v_{k}^{i}\right| \\
& =\left|S_{2} g_{0}\left(e_{k}^{1}\right)\left(e_{k}^{i}-e_{k}^{1}\right)\right| \leq C \rho^{2}\left(e_{k}^{i}, e_{k}^{1}\right)|| g_{0}|| \\
& \leq 4 C \delta^{2} C\left(A^{3}, \theta\right)\|w\|_{\mathcal{V}^{1}(B)}
\end{aligned}
$$

where $C$ is an absolute constant given by Lemma 7 .

For $I I$, remark that the second supremum has to be taken on $e_{k}^{i} \in B$ and $e_{p}^{j} \in B \cap K\left(e_{k}^{i}, \delta\right)$ but the fact that packets of $B$ are $\delta$-separated implies that $B \cap K\left(e_{k}^{i}, \delta\right) \subset G_{k}$ and this implies $p=k$. We use the same 
method as in $I$ and obtain

$$
\begin{aligned}
\left|\frac{v_{k}^{i}-g_{0}\left(e_{k}^{i}\right)-\left(v_{k}^{j}-g_{0}\left(e_{k}^{j}\right)\right)}{\rho\left(e_{k}^{i}, e_{k}^{j}\right)}\right| & =\left|\frac{S_{2} g_{0}\left(e_{k}^{1}\right)\left(e_{k}^{i}-e_{k}^{1}\right)-S_{2} g_{0}\left(e_{k}^{1}\right)\left(e_{k}^{j}-e_{k}^{1}\right)}{\rho\left(e_{k}^{i}, e_{k}^{j}\right)}\right| \\
& \leq C\left\|g_{0}\right\| \frac{\rho^{2}\left(e_{k}^{i}, e_{k}^{1}\right)+\rho^{2}\left(e_{k}^{j}, e_{k}^{1}\right)}{\rho\left(e_{k}^{i}, e_{k}^{j}\right)} .
\end{aligned}
$$

And by the angle condition $\frac{\rho\left(e_{k}^{j}, e_{k}^{1}\right)}{\rho\left(e_{k}^{i}, e_{k}^{j}\right)} \leq \frac{1}{\sin \theta}$ so

$$
I I \leq 4 C\left\|g_{0}\right\| \frac{\delta}{\sin \theta} \leq 4 C \cdot C\left(A^{3}, \theta\right) \frac{\delta}{\sin \theta} .
$$

To estimate $I I I$ we use the estimation of $I$ together with $\rho\left(e_{p}^{j}, e_{k}^{i}\right) \geq \delta$. We obtain

$$
I I I \leq 8 C \delta C\left(A^{3}, \theta\right)\|w\|_{\mathcal{V}^{1}(B)} .
$$

So finally there exists a constant $M=16 C \cdot \frac{C\left(A^{3}, \theta\right)}{\sin \theta}$ depending only on $\theta$ and $A^{3}$ such that

$$
\left\|w^{1}\right\|_{\mathcal{V}^{1}(B)} \leq M \delta\|w\|_{\mathcal{V}^{1}(B)} .
$$

We choose $\delta$ such that $M \delta<1$ and we apply again the same process; we construct a sequence of functions $\left(g_{k}\right)_{k \in \mathbb{N}} \in H^{\infty}$ and $\left(w^{k}\right)_{k \in \mathbb{N}}$ sequences of $\mathcal{V}^{1}(B)$ satisfying

$$
\left\{\begin{array}{l}
w^{k}=w^{k-1}-g_{k-1 \mid B}, \\
\left\|w^{k}\right\|_{\mathcal{V}^{1}(B)} \leq(M \delta)^{k}\|w\|_{\mathcal{V}^{1}(B)}, \\
\left\|g_{k}\right\| \leq M\left\|w^{k}\right\|_{\mathcal{V}^{1}(B)} .
\end{array}\right.
$$

Define $f=\sum_{q=0}^{\infty} g_{q}$, then $f$ is solution of the interpolation problem (14). Indeed the series for $f$ converges normally and

$$
\begin{aligned}
\left\|f_{\mid B}-w\right\|_{\mathcal{V}^{1}(B)} & =\|\sum_{q=1}^{\infty} g_{q}+\underbrace{g_{0}-w}_{w^{1}}\|_{\mathcal{V}^{1}(B)}, \\
& =\cdots=\left\|\sum_{q=N}^{\infty} g_{q}+w^{N}\right\|_{\mathcal{V}^{1}(B)} \quad \text { where } N \in \mathbb{N}, \\
& \leq\left\|\sum_{q=N}^{\infty} g_{q}\right\|_{\mathcal{V}^{1}(B)}+\left\|w^{N}\right\|_{\mathcal{V}^{1}(B)},
\end{aligned}
$$


and this last quantity tends to 0 when $N$ goes to infinity, we deduce $f_{\mid B} \equiv w$ and so $B=B^{3}$ is an interpolating sequence of order 1 . By the same method one shows that $B^{1}, B^{2}$ are interpolating sequences too, and the Proposition 15 allows us to conclude that $Z=B^{1} \cup B^{2} \cup B^{3}$ is an interpolating sequence of order 1 .

\section{References}

[Ber71] A. Bernard, Algèbre quotient d'algèbre uniforme, C. $R$. Acad. Sci. Paris Sér. A-B 272 (1971), 1101-1104.

[Ber85] B. Berndtsson, Interpolating sequences for $H^{\infty}$ in the ball, Nederl. Akad. Wetensch. Indag. Math. 47(1) (1985), 1-10.

[BNØ96] J. Bruna, A. Nicolau And K. Øyma, A note on interpolation in the Hardy spaces of the unit disc, Proc. Amer. Math. Soc. 124(4) (1996), 1197-1204.

[Car58] L. CARLESON, An interpolation problem for bounded analytic functions, Amer. J. Math. 80 (1958), 921-930.

[Dru72] S. W. Drury, Unions of sets of interpolation, in "Conference on Harmonic Analysis" (Univ. Maryland, College Park, Md., 1971), Lecture Notes in Math. 266, Springer, Berlin, 1972, pp. 23-33.

[Gar81] J. B. Garnett, "Bounded analytic functions", Pure and Applied Mathematics 96, Academic Press Inc., New York, 1981.

[Har99] A. HaRTMAnN, Traces of certain classes of holomorphic functions on finite unions of Carleson sequences, Glasg. Math. J. 41(1) (1999), 103-114.

[Hir80] J.-B. Hiriart-Urruty, Extension of Lipschitz functions, $J$. Math. Anal. Appl. 77(2) (1980), 539-554.

[Mar98] N. Marco, Interpolation dans l'espace de Hardy du disque, Prépublication UPS, no. 107 (1998).

[McS34] E. J. McShane, Extension of range functions Lipschitz functions, Bull. Amer. Math. Soc. 40 (1934), 837-842.

[Nik78a] N. K. NIKOL'SKIĬ, Bases of invariant subspaces and operator interpolation, Trudy Mat. Inst. Steklov. 130 (1978), 50-123, 223.

[Nik78b] N. K. NIKOL'SKII , What problems do spectral theory and complex analysis solve for each other?, in: "Proceedings of the International Congress of Mathematicians" (Helsinki, 1978), Acad. Sci. Fennica, Helsinki, 1980, pp. 631-638. 
[Rud80] W. RudiN, "Function theory in the unit ball of $\mathbb{C}^{n}$ ", Grundlehren der Mathematischen Wissenschaften 241, Springer-Verlag, New York, 1980.

[SS61] H. S. Shapiro AND A. L. ShIElds, On some interpolation problems for analytic functions, Amer. J. Math. 83 (1961), 513-532.

[Tho97] P. J. Thomas, Necessary conditions for interpolating sequences, Bull. London Math. Soc. 29(4) (1997), 433-442.

[Var71] N. T. VAropoulos, Sur la réunion de deux ensembles d'interpolation d'une algèbre uniforme, C. R. Acad. Sci. Paris Sér. $A-B 272$ (1971), A950-A952.

[Var72] N. T. Varopoulos, Sur un problème d'interpolation, $C$. $R$. Acad. Sci. Paris Sér. A-B 274 (1972), A1539-A1542.

[Vas79] V. I. VAsJunin, Unconditionally convergent spectral decompositions and interpolation problems, Trudy Mat. Inst. Steklov. 130 (1978), 5-49, 223.

Université Paul Sabatier

Laboratoire Emile Picard

UFR MIG

118 route de Narbonne

31063 Cedex Toulouse

France

E-mail address: nmarco@picard.ups-tlse.fr

Primera versió rebuda el 19 de setembre de 2000 , darrera versió rebuda el 13 d'octubre de 2000. 